\title{
Flame Propagation in a Gasification Channel
}

\author{
Dmitry N. Saulova ${ }^{\mathrm{a}}$ Ovid A. Plumb ${ }^{\mathrm{b}}$, A.Y. Klimenko*,a \\ ${ }^{a}$ School of Engineering, The University of Queensland, St. Lucia, QLD 4172, \\ Australia \\ ${ }^{b}$ College of Engineering and Applied Science, University of Wyoming, Laramie, WY \\ 82071, USA
}

\begin{abstract}
In the present study, propagation of a gasification flame through a coal channel is considered. A simplified physical model, which nevertheless incorporates all main physical factors determining the flame front propagation in a gasification reactor, is suggested. As demonstrated, the flame propagation is governed by energy balance in the channel. The suggested model is in an agreement with experimental observations obtained in underground gasification of coal (UCG).
\end{abstract}

Key words: gasification channel, energy balance, flame propagation

\section{Introduction}

Flame front propagation, which is an important issue in many technological problem in power generation, petrochemical and others industries, has received substantial attention over the years (see, for example, Refs. [1-7]). As pointed out by Chernyshev [8] and Skafa [9], the position and propagation of flames in the gasification channel are of great importance for sustainable operations in underground coal gasification (UCG). Correct positioning of the flame between the injection and production wells is a significant factor affecting success of operations during the link enhancement and main gasification stages. Here, we introduce a theory that takes into account the energy balance in a gasification channel and explains dependence of the flame speed on the air injection rate, which represents the primary control parameter, and other parameters of the flow. This paper focuses on the physics of the phenomenon, while specific UCG operational procedures remain outside the scope of this work.

The complexity of the problem of premixed flame propagation in turbulent flows is well known [4]. The majority of the previous studies on the flame front propagation concentrated on homogeneous reactions in premixed mixtures either in laminar [6] or turbulent [5] flows. The channel walls were considered to be made of inert materials and were assumed to be adiabatic [3], or the outer surface of the wall was assumed to be maintained at the constant ambient

\footnotetext{
*Corresponding author, E-mail: a.klimenko@uq.edu.au
} 
temperature [1-3]. In the latter case, the influence of the heat losses to the channel walls on the flame propagation was investigated. Previous studies were also concentrated on the cases, in which the conduction of heat through the gas phase is one of the major mechanisms of heat transfer in the axial direction of the channel.

In contrast to the previous studies, we consider propagation of the flame front in the context of gasification of coal in a channel which, in UCG conditions, is characterized by high velocities of the gas flow. In this case, the conduction of heat through the gas flow in the axial direction of the channel is negligibly small compared to other heat transfer mechanisms, such as radiation, gas convection and conduction through the channel walls. Another distinction of the gasification flame from the previously studied cases, where the channel walls are considered to be non-reactive and non-catalatic, is the importance of heterogeneous reactions between gas and the channel walls (coal).

Various aspects of coal gasification technologies and UCG in particular (see, for example, Refs. [10-19]) have been repeatedly studied over the years. CFD modelling of reacting flows is used in majority of these studies. Principal description and analysis of the propagation of the gasification flame is given by Skafa [9]. However, no analytical results governing position and propagation of the gasification flame can be found in the literature. In this paper we suggest a physical approach, which explains the main features of the flame behavior in a channel based on first principles and offers an approximate analytical expression for the flame speed. To develop this physical model, we make certain simplifying assumptions, which allow for an analytical characterization but retain physical effects that are of primary importance to the phenomenon in question. The model predictions are in good qualitative agreement with the experimental evidence from practical UCG operations [8, 9].

\section{Heat transfer in the radial direction}

The strategy of this work is in simplifying the problem as much as it possible, while retaining major physical factors for consideration. The solutions are then generalized to take into account additional factors that may be of prime importance under certain conditions. Whenever possible, we wish to obtain a steady or quasi-steady solution for the problem. This preference is understandable since the flame front evolves slowly compared to the velocities present in the air flow.

We consider an infinitely long channel through the block of a combustible material.Initially, the material is at the ambient temperature. After some time from the initiation of combustion, a layer of some thickness surrounding the part of the channel with product gas will be heated up to the temperature of the gas. The thickness of such a layer increases in time. The rate of this increase, however, rapidly declines. To model this, we assume an existence of the adiabatic wall over a layer of coal of thickness $\delta$ surrounding the channel.

This assumption is even more justifiable in the case of UCG, where the gasification channel is, usually, below the water table. To prevent contamina- 
tion of local aquifer by product gas, the operational pressure in the channel is maintained below the hydrostatic pressure in environmentally responsible UCG operations [20, 21]. This pressure difference produces influx of ground water into the channel and prevents product gas from leaving the reactor. Apart from preventing aquifer contamination, the water influx also prevent enthalpy losses to the surroundings. The enthalpy conducted and irradiated from the reactor is used for heating and evaporating incoming water and for heating the incoming steam up to the reactor temperature. As a result, the reactor is covered by a layer of steam, which is called, according to terminology introduced by Blinderman et al. [20, 21], the "steam jacket". The enthalpy lost in conduction and radiation returns to the reactor in a form of sensible enthalpy of the incoming steam. The issue of stability of the steam jacket is analyzed in Ref. [22]. For the purposes of this paper, however, we neglect the incoming water as an independent phase and treat it as contained in coal, while considering the thermodynamic balance.

\section{Radiation in a Cavity}

Including the effect of radiation when analyzing transport phenomena in a cavity presents challenges unless a diffusion approximation can be used so that radiation can be evaluated utilizing the same differential equations as in the analysis of transport by convection and diffusion.

The diffusion approximation for radiation can be justified by considering that the gasification flame has a significant extent along the channel. Here, the channel is treated as an infinite cylinder having radius $R$ extending from $x=0$ to $x=+\infty$ in one direction and to $x=-\infty$ in the other direction. The location $x=0$ is selected for simplicity and, of course, can represent any physical location along the cylinder. The cylinder surface temperature $T(x)$ is allowed to vary (gradually) with $x$ and the cylinder is filled with a transparent medium.

The heat flowing down the tube from $x>0$ is given by

$$
q_{(+)}=2 \pi R \sigma \int_{0}^{+\infty} F_{d 1-0}\left(\frac{x}{D_{R}}\right) T^{4}(x) d x .
$$

Where $F_{d 1-0}$ is the radiation view factor from a differential ring $d 1$ on a cylinder to the cylinder's base at $x=0$ given by $[23,24]$

$$
F_{d 1-0}(X)=\frac{X^{2}+1 / 2}{\sqrt{1+X^{2}}}-X,
$$

where $X=x / D_{R}, D_{R}=2 R$ is the channel diameter, the differential ring is of area $d S=2 \pi R d x$ and located at a distance $x$ from the base of the cylinder of radius, $R$, and base area is given by $S_{0}=\pi R^{2}$. Similarly, the radiant flux from the section of the tube $x<0$ is given by

$$
q_{(-)}=2 \pi R \sigma \int_{0}^{-\infty} F_{d 1-0}\left(\frac{x}{D_{R}}\right) T^{4}(x) d x .
$$


We note that the view factors $F_{d 1-0}$ in Eqs. (1) and (3) are the same due to symmetry of the cylinder. Where surface 1 is the differential tube wall located at $x=x_{1}$. Surface 0 is the circular disc at $x=0$. The overall radiant heat flux is given by the difference

$$
q_{\mathrm{rad}}=q_{(+)}-q_{(-)} .
$$

If we expand the forth power of temperature as a Taylor series

$$
T(x)^{4}=\left(T^{4}\right)_{x=0}+4\left(T^{3}\right)_{x=0}\left(\frac{d T}{d x}\right)_{x=0} x+\ldots
$$

the integral takes the form

$$
q_{\mathrm{rad}}=64 \pi R^{3} \sigma T^{3} \frac{d T}{d x} \int_{0}^{\infty} X F_{d 1-0}(X) d X=\frac{32}{3} \pi R^{3} \sigma T^{3} \frac{d T}{d x} .
$$

In this equation, the integral is evaluated as $1 / 6$. This integral does not converge extremely fast so the analysis is restricted to a long cylinder with small radius. For example, at $X=15$ the value of the integral is $95 \%$ of its value $(1 / 6)$ at $X=\infty$ and it reaches $99 \%$ of the value at infinity when $X=50$. It is about $90 \%$ of $1 / 6$ at $X=7$. In this work, we use this equation for estimates of the effective conductivity coefficient and this accuracy is sufficient. It should be also noted that linear approximation used here for the temperature would tend to overpredict $q_{\mathrm{rad}}$ when temperature disturbances are rapid $\Delta x<D_{R}=2 R$ where $\Delta x$ is the length of the disturbance. For example, if we consider a small increment of temperature $\Delta T$ that is stretched over small distance $\Delta x$, the radiative flux would tend to a finite value

$$
q_{\mathrm{rad}}=32 \pi R^{2} \sigma T^{3} \Delta T \int_{0}^{\infty} F_{d 1-0}(X) d X=8 \pi R^{2} \sigma T^{3} \Delta T
$$

as $\Delta x \rightarrow 0$. The approximation of the flux in Eq. (6) is given by $q_{\mathrm{rad}}=$ $\frac{32}{3} \pi R^{3} \sigma T^{3}(\Delta T / \Delta x) \rightarrow \infty$ as $\Delta x \rightarrow 0$. Comparing these equations indicates that overestimation of $q_{\mathrm{rad}}$ occurs for $\Delta x<\frac{4}{3} R=\frac{2}{3} D_{R}$.

The final equation for the heat flux per area takes the form

$$
\dot{h}=-\lambda_{\mathrm{rad}} \frac{d T}{d x}
$$

where

$$
\lambda_{\mathrm{rad}}=\frac{16}{3} \sigma D_{R} T^{3}
$$

is the effective the radiative conductivity $(\mathrm{W} / \mathrm{m}-\mathrm{K}), \dot{h}$ is the heat flux (per unit area), $\sigma=5.67 \mathrm{E}-8 \mathrm{~W} \cdot \mathrm{m}^{-2} \cdot \mathrm{K}^{-4}$ is the Stefan-Botzmann constant, $D_{R}=2 R$ is the cavity diameter and $T$ is the absolute temperature of the cavity wall. This equation can also be approximately applied to an irregular fracture of width $D_{R}$. 


\section{Model description}

\subsection{Governing equations}

Transport and reactions in a channel are governed by the equations of conservation of mass

$$
\frac{\partial \rho}{\partial t}+\nabla \cdot(\rho \mathbf{v})=0
$$

species

$$
\frac{\partial \rho_{i}}{\partial t}+\nabla \cdot\left(\rho_{i} \mathbf{v}_{i}\right)=W_{i}
$$

and energy

$$
\frac{\partial \rho h}{\partial t}+\boldsymbol{\nabla} \cdot(\rho \mathbf{v} h)-\boldsymbol{\nabla} \cdot(\lambda \boldsymbol{\nabla}(T))=0 .
$$

Here, $\mathbf{v}_{i}, \rho_{i}$ and $W_{i}$ are the velocity, density (mass per volume) and the chemical reaction source term of the $i$ 'th component, respectively. The subscript index " $i$ " runs over all species of interest. The overall density is denoted by $\rho$, so that the mass fraction $Y_{i}$ of the $i$ th component is given by $Y_{i}=\rho_{i} / \rho$ and $\mathbf{v}$ is the overall velocity in the selected frame of reference. The diffusion coefficient, which includes both the heat conduction term $\lambda_{\text {cond }}$ and the radiation term $\lambda_{\text {rad }}$ given by Eq. (9), is denoted by $\lambda=\lambda_{\text {cond }}+\lambda_{\text {rad }}$. Under practical conditions, the radiative term completely dominates the conductive term. The temporal pressure derivative $\partial p / \partial t$ is conventionally neglected in the energy equation.

Now consider a flame propagating in a cylindrical channel and use the frame of reference attached to the moving flame so that $x$-axis is normal to the flame front with the positive direction coincides with that of gas stream. The $y$ and $z$ axes are parallel to the flame front. We assume that there are no fluxes and heat losses in the radial direction (see Section 2). We also assume the existence of steady state solution. Integrating Eqs. (10-12) over $(y-z)$-plane within the limits of control volume $R \leq R_{\mathrm{cv}}$ one obtains

$$
\begin{gathered}
\frac{\partial \bar{m}}{\partial x}=0, \\
\frac{\partial \overline{m_{i}}}{\partial x}=\overline{W_{i}}, \\
\frac{\partial \overline{m h}}{\partial x}-\frac{\partial}{\partial x}\left(\overline{\left.\lambda \frac{\partial T}{\partial x}\right)=0,}\right.
\end{gathered}
$$

Here $\bar{m} \equiv \overline{\rho v}$ and $\overline{m_{i}} \equiv \overline{\rho_{i} v_{i}}$ are mass flow rates, where $v$ is the $x$-component of the velocity vector $\mathbf{v}$. Over-bars denote integration over the $(y-z)$-plane within the control volume:

$$
\overline{(\cdot)}=\int_{\substack{\text { C.V. } \\ x=\text { const }}}(\cdot) d y d z
$$


Variations of the the temperature in the radial direction is neglected as well as radial variations of the other parameters within a selected phase (gas or solid). That is,

$$
\overline{\lambda \frac{\partial T}{\partial x}}=\bar{\lambda} \frac{\partial T}{\partial x}, \quad(\overline{m h})_{i}=\bar{m}_{i} h_{i},
$$

etc, assuming that each of species " $i$ " is present only in a single phase. With the use of sensible enthalpies $h^{(s)}$, Eq. (15) takes the form

$$
\frac{\partial \overline{m h^{(s)}}}{\partial x}-\frac{\partial}{\partial x}\left(\bar{\lambda} \frac{\partial T}{\partial x}\right)=\bar{W}_{h}
$$

where $\bar{W}_{h}$ is the heat source which is determined in the following sections.

\subsection{Reaction kinetics}

The reaction mechanism in the oxidation zone is complex and includes both heterogeneous (gas-solid interface) and homogeneous (gas phase) reactions. It may also include phase transitions (evaporation of water or coal volatile matter, for example). This mechanism, however, can be represented as the following generalized reaction

$$
\mu_{g}^{-}(\mathrm{Gas})^{-}+\mu_{s}^{-}(\mathrm{Solid})^{-} \rightarrow \mu_{g}^{+}(\mathrm{Gas})^{+}+\mu_{s}^{+}(\mathrm{Solid})^{+} .
$$

In terms of species the above reaction takes the form

$$
\sum_{i} \mu_{i}^{-}(\text {Species })_{i} \rightarrow \sum_{i} \mu_{i}^{+}(\text {Species })_{i}
$$

Here and later in the paper the superscripts "-" and "+" denote values before and after the reaction, respectively. The stoichiometric coefficients $\mu_{i}$ are on the mass basis and $\mu_{i}^{+}-\mu_{i}^{-}$represents the mass change of $i^{\text {th }}$ component. In the rest of the paper we put $\mu_{g}^{-}=1$ without loss of generality. The global reaction is presumed to be specified so that the product coefficients depend on $\mu_{s}^{-}$so that $\mu_{i}^{ \pm}=\mu_{i}^{ \pm}\left(\mu_{s}^{-}\right)$for any component " $i$ " (or phase " $i$ "). All of the reactions are presumed to form a global reaction that has a single rate so that we can write for any $i$ and $j$

$$
\frac{\bar{W}_{i}}{\mu_{i}^{+}-\mu_{i}^{-}}=\frac{\bar{W}_{j}}{\mu_{j}^{+}-\mu_{j}^{-}} .
$$

The rate of oxygen consumption $\bar{W}_{\mathrm{O}_{2}}$ in the reaction (19) is given by the equation

$$
\bar{W}_{\mathrm{O}_{2}}=-\rho_{\mathrm{O}_{2}} \bar{A} K(T) \text {. }
$$

Here, $\rho_{\mathrm{O}_{2}}$ is density of oxygen, $K(T)$ is a function of temperature and $\bar{A}$ is the reaction pre-exponential factor. The consumption of oxygen from the injected gas (air) mainly occurs on the channel walls. There are two major factors limiting the consumption of oxygen: the combined rate of heterogeneous reactions, 
which exponentially depends on temperature, and the diffusive flux of oxygen to the channel walls. To account for these factors, we assume that

$$
\frac{1}{K(T)}=\frac{1}{K_{r}(T)}+\frac{1}{K_{d}}
$$

where $K_{d}$ is a constant, while $K_{r}(T)$ is given by

$$
K_{r}(T)=\exp \left(-T_{E} / T\right)
$$

Here, $T_{E}$ is the activation temperature.

In Eq. (23), the constant $K_{d}$ describes the the transport-limited regime and the function $K_{r}(T)$ is related to the reaction-limited regime. For temperatures lower than $T_{d}$, which is given by $T_{d}=-T_{E} / \ln \left(K_{d}\right)$, the rate is reaction-limited and $K \approx K_{r}$. For the temperatures higher than $T_{d}$, the rate is transport-limited and $K \approx K_{d}=\exp \left(-T_{E} / T_{d}\right)$. Note that all kinetic parameters introduced here are effective parameters that are conventionally used to approximate the average reaction rate.

\subsection{Integrals across the flame}

There are two possible regimes of combustion: reverse combustion and forward combustion (see, for example, Ref [25] for more details). In the former case, the combustion temperature equals to the adiabatic temperature for a given fuel/air ration. In the latter case, the combustion temperature exceeds the adiabatic one. This superadiabatic effect occurs due to the fact that either oxidant or fuel enters the reaction zone at elevated temperature. In the case of forward combustion the travelling heat wave can generally have either reaction leading or reaction trailing structure. As discussed in Ref [25], however, the case of forward combustion with the reaction leading structure is not possible in practical UCG conditions. For this reason, we exclude the reaction leading structure from our consideration. We also exclude the fuel deficient branch of the reaction trailing structure, since oxidant and coal can not coexist at high temperature and coal is always present in the channel.

Integration of Eqs. (13-15) across the flame yields

$$
\begin{gathered}
\bar{m}_{u}=\bar{m}_{b} \\
\bar{m}_{i, b}=\bar{m}_{i, u}+Q_{i}, \\
(\bar{m} h)_{b}=(\bar{m} h)_{u}
\end{gathered}
$$

Here, the subscript " $u$ " (unburned) is used to denote values upstream from the flame front, while the subscript " $b$ " (burned) denotes values downstream from the flame front as depicted in Fig. 1.

In Eq. (26), $Q_{i}$ are given by

$$
Q_{i} \equiv \int_{u}^{b} \bar{W}_{i} d x=\int_{-\infty}^{+\infty} \bar{W}_{i} d x
$$




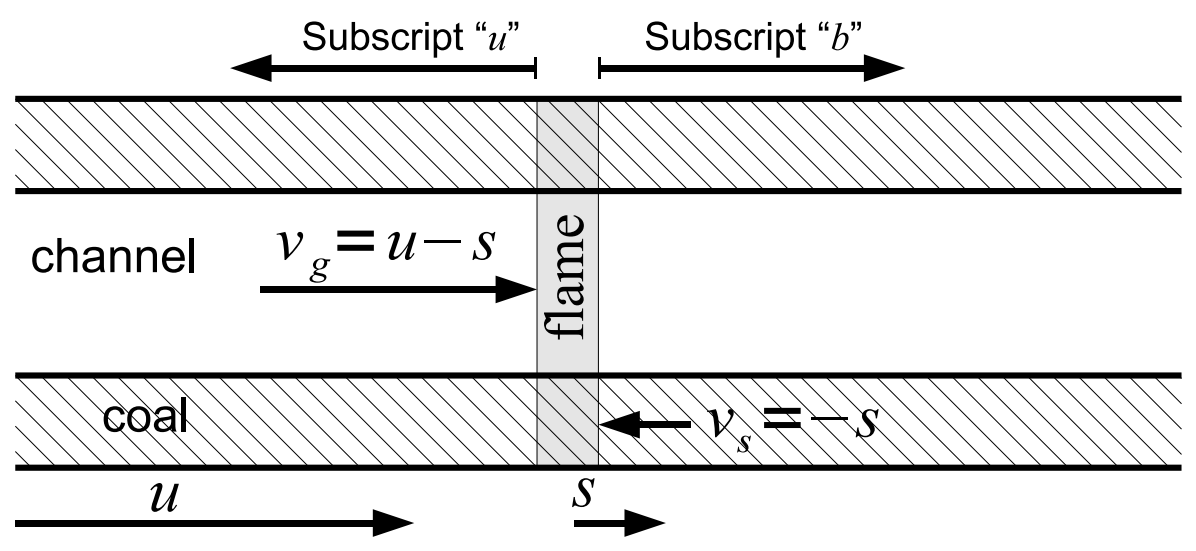

Figure 1: Schematic view of the gasification channel.

The analysis of the enthalpy equation (15) can be performed similar to that in Ref. [26] by representing the total enthalpy $h_{i}$ of any component " $i$ " as a sum of sensible enthalpy $h_{i}^{(s)}$ and the enthalpy of formation $h_{i}^{(f)}$ (see Ref. [26] for more details). The overall heat released in the reactions is

$$
Q_{h} \equiv \int_{-\infty}^{+\infty} \bar{W}_{h} d x=\bar{m}_{g, u} H\left(\mu_{s}^{-}\right) .
$$

Here, $\bar{W}_{h}=\theta \bar{W}_{\mathrm{O}_{2}}$ is the enthalpy source and $\bar{m}_{g, u}$ is the mass flow rate of unburned gas. The enthalpy of the reaction $H\left(\mu_{s}^{-}\right)$is given by

$$
H\left(\mu_{s}^{-}\right) \equiv \sum_{i}\left(\mu_{i}^{-}-\mu_{i}^{+}\right) h_{i}^{(f)}
$$

and

$$
\theta \equiv \frac{H\left(\mu_{s}^{-}\right)}{\mu_{\mathrm{O}_{2}}^{-}-\mu_{\mathrm{O}_{2}}^{+}} .
$$

\subsection{The released heat}

We conventionally use the traditional Zeldovich high activation energy asymptotics [27] to approximate the overall heat release. These equations were modified slightly to account for reduced influence of upstream diffusion of oxygen in gasification conditions - the details are given in Ref. [26], while the mathematical approach used in this work is presented in Refs. [7, 28]. Two usual zones are distinguished in the flame: the preheat zone, where reactions are not intense, and the reaction zone, where reactions are most intense. Note that in a gasification flame these zones are followed by the reduction zone, which has a lesser effect on the flame speed. The equations for the heat release in the 
reaction zone are written as $[26]$

$$
Q_{h}\left(T_{b}, \ldots\right)=\left(\bar{\lambda} \frac{\partial T}{\partial x}\right)_{(-r)}=\sqrt{\left(\rho_{O 2}\right)_{0} \bar{A} \theta \bar{\lambda}_{r} \Phi\left(T_{b}\right)}
$$

where the subscript " $-r$ " denotes a value just upstream the reaction zone (i.e. between the reaction zone and preheat zone), $\bar{\lambda}_{r}$ is the value of $\bar{\lambda}$ in the reaction zone, $\left(\rho_{\mathrm{O} 2}\right)_{0}$ is the initial mass concentration of oxygen and $\Phi\left(T_{b}\right)$ is given by

$$
\Phi\left(T_{b}\right)=\int_{-\infty}^{T_{b}} K(T) d T=\frac{K_{d}}{Z} \ln \left(1+\frac{K_{r}\left(T_{b}\right)}{K_{d}}\right),
$$

In Eq. (33), $\bar{A}, K(T), K_{r}$ and $K_{d}$ have the same meaning as in Eqs. (22) and (23), $Z \equiv T_{E} / T_{b}{ }^{2}$.

\subsection{The velocity of the flame front}

Let $u$ be the absolute velocity of gas and $s$ be the flame front velocity in a stationary system of coordinates (see Fig. 1). The velocity of gas $v_{g}$ and solid $v_{s}$ phases in the moving frame of reference (attached to the flame front) is given by

$$
v_{g}=u-s, \quad v_{s}=-s .
$$

Note that the value of $s$ is positive, when the flame propagates downstream. In the opposite case, the value of $s$ is negative. Typically, $u$ is much higher than $s$ and the difference between $u$ and $v_{g}$ can be neglected for all practical purposes.

Similar to the previous paper [26], the flame front velocity can be obtained without specific analysis of the preheat zone. Recalling that $\mu_{g}^{-}=1$, one verifies that for any $i$ representing different species or phases the reaction coefficients $\mu_{i}$ are related to the mass flow rates by the following equations

$$
\mu_{i}^{+}=\frac{\bar{m}_{i}^{+}}{\bar{m}_{g}^{-}}, \mu_{i}^{-}=\frac{\bar{m}_{i}^{-}}{\bar{m}_{g}^{-}} .
$$

Here, the superscript "-" denotes the mass flow rates of the reactants entering the reaction zone, while the superscript "+" denote the mass flow rates of products leaving the reaction zone. Note that all $\bar{m}_{i}^{-}$and $\bar{m}_{i}^{+}$are always nonnegative, while mass flow rates of solid species denoted by subscripts " $u$ " and " $b$ " are negative in the case of forward combustion. That is, $\bar{m}_{s}^{-}=-\bar{m}_{s, b}$ and $\bar{m}_{s}^{+}=-\bar{m}_{s, u}$, in the case of forward combustion, while $\bar{m}_{s}^{-}=\bar{m}_{s, u}$ and $\bar{m}_{s}^{+}=\bar{m}_{s, b}$, in the opposite case. For gaseous species, $\bar{m}_{g}^{-}=\bar{m}_{g, u}$ and $\bar{m}_{g}^{+}=\bar{m}_{g, b}$ in both cases.

The enthalpy equation (27), can be expressed in terms of $h_{i}$ and divided by $\bar{m}_{g, u}$, determines the temperature of the products $T_{b}$ as a function of fuel/air ratio $\mu_{s}^{-}$and other parameters. That is, $T_{b}=T_{b}\left(\mu_{s}^{-}, \ldots\right)$.

Using Eq. (35), neglecting the variation of $\rho_{s}$ across the flame and taking into account that $\rho_{s} \gg \rho_{g}$, while $\mu_{s}^{-}$is of the order of unity, one can obtain

$$
s= \pm \frac{\bar{m}_{s}^{-}-\bar{m}_{s}^{+}}{\rho_{s} S_{\mathrm{cons}}}
$$


where

$$
\bar{m}_{s}^{-}-\bar{m}_{s}^{+}=\left(\mu_{s}^{-}-\mu_{s}^{+}\right) \bar{m}_{g}^{-}, \quad \bar{m}_{g}^{-}=\frac{Q_{h}\left(T_{b}, \ldots\right)}{H\left(\mu_{s}^{-}\right)} .
$$

In Eq. (36), $S_{\text {cons }}$ is the area occupied by coal burned by the flame as it enters the control volume and the sign "+" corresponds to the case of forward combustion, while sign "-" is used in the case of reverse combustion.

\subsection{The energy balance equation for a cylindrical channel}

As discussed in Section 2, we assume that there is a hot layer of coal (at temperature $T_{b}$ ) of thickness $\delta$ surrounding the cylindrical gasification channel downstream from the flame front. The thickness $\delta$ is an adjustable parameter of the model that is related to the thickness of the "steam jacket". The rest of coal is assumed to be at the ambient temperature $T_{u}$. The velocity of the flame front depends on the temperature of the products $T_{b}$, which, in its turn, depends on the fuel/air ratio $\mu_{s}^{-}$and the parameter $\delta$. The functions $T_{b}\left(\mu_{s}^{-}, \delta, \ldots\right)$ and $Q_{h}\left(T_{b}, \ldots\right)$ also depend on other parameters, such as $T_{u}, \bar{A}, T_{E}$, and initial compositions of coal and air that are fixed and selected to match the conditions in practical UCG operations. In order to elaborate the functions $T_{b}\left(\mu_{s}^{-}, \delta, \ldots\right)$, we consider reverse combustion (the flame front propagates upstream) and forward combustion (the flame front propagates downstream). We assume that the density of coal, its heat capacity and velocity do not change across the flame. That is, $\rho_{s, u}=\rho_{s, b}=\rho_{s}, h_{s, u}(T)=h_{s, b}(T)=h_{s}(T)$ and $v_{s, u}=v_{s, b}=v_{s}$.

The case of reverse combustion is schematically depicted in Fig. 2(a), where the control volume of the radius $R_{\mathrm{cv}}=R_{b}+\delta$ is presented as the shaded area. As can be seen in this figure, air and coal enter the control volume at the ambient temperature $T_{u}$. Unreacted coal and product gas leave the control volume at the temperature $T_{b}$. In the case of reverse combustion, $T_{b}$ is the adiabatic temperature.

The schematic view of forward combustion, when $R_{b}+\delta \leq R_{u}$ is given in Fig. 2(b). In this case, we select the control volume of the radius $R_{\mathrm{cv}}=R_{u}$. As shown in Fig. 2(b), some part of coal entering the control volume is preheated to the temperature $T_{b}$. The other part of coal entering the control volume is at the ambient temperature. Note that all coal reacts in the control volume. Air enters the control volume at the ambient temperature, while product gas leaving it at the temperature $T_{b}$. The burned temperature $T_{b}$ is superadiabatic, since part of coal entering the reaction zone is preheated.

Now consider the case of forward combustion, when $R_{b}+\delta>R_{u}$. As shown in Fig 2(c), we select the control volume of the radius $R_{\mathrm{cv}}=R_{b}+\delta$. All coal enters the control volume at $T_{b}$, while air enters the control volume at the ambient temperature. Unreacted coal leaves the control volume at ambient temperature $T_{u}$, while product gas leaves it at the temperature $T_{b}$. Note that $T_{b}$ is superadiabatic and even higher than in the previous case, since all coal entering the reaction zone is preheated.

The heat balance equation (27) reads

$$
\left(\bar{m}_{s} h_{s}\right)_{u}+\left(\bar{m}_{g} h_{g}\right)_{u}=\left(\bar{m}_{s} h_{s}\right)_{b}+\left(\bar{m}_{g} h_{g}\right)_{b} .
$$




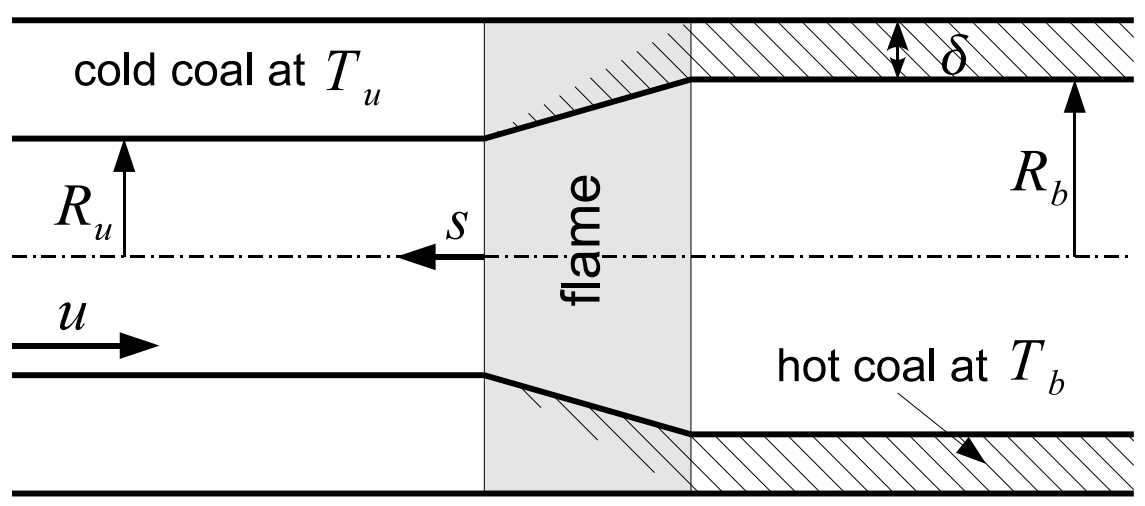

(a)

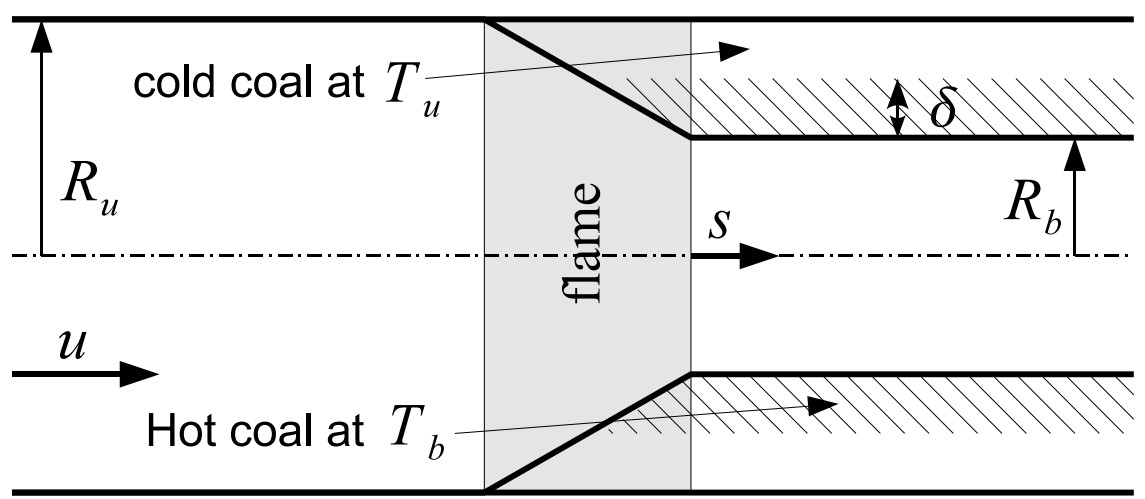

(b)

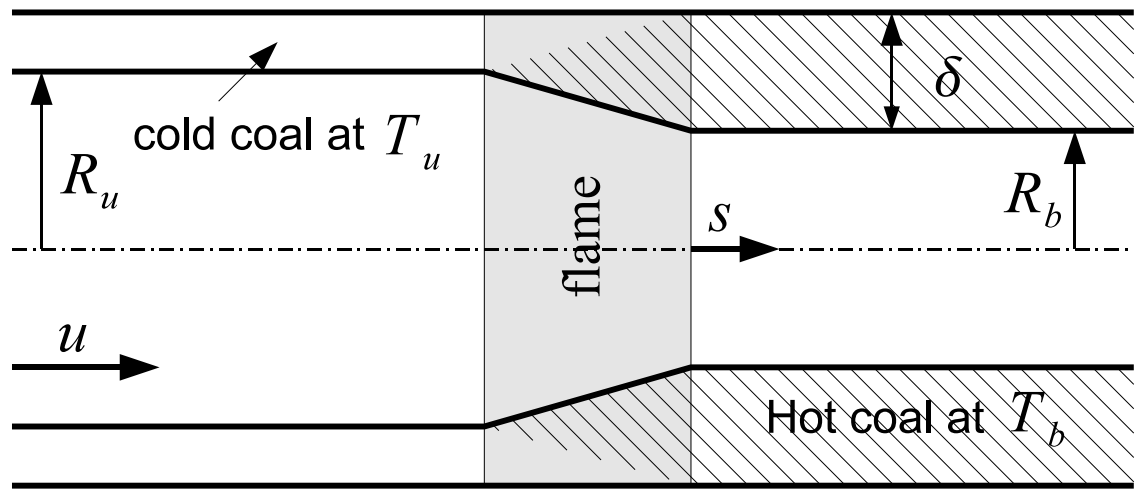

(c)

Figure 2: Schematic representation of flame propagation regimes in cylindrical gasification channel. (a) - Reverse combustion. (b) - Forward combustion in cylindrical gasification channel when $R_{b}+\delta \leq R_{u}$. (c) - Forward combustion in cylindrical gasification channel when $R_{b}+\delta>R_{u}$ 
Note that the velocity of coal $v_{s}$ and the radii difference $\left(R_{b}-R_{u}\right)$ are negative, when flame propagates downstream, and are positive otherwise. If known, the heat losses can be easily accommodated in Eq. (38). In the case depicted in Fig. 2(b), only some part of the coal enters the control volume preheated, while the rest of coal enters the control volume at the ambient temperature. Then, Eq. (38), being written for the appropriate control volume, takes the following form in all three cases considered above.

$$
\begin{aligned}
& v_{s} \rho_{s} \pi\left(\left(R_{b}+\delta\right)^{2}-R_{u}^{2}\right) h_{s}\left(T_{u}\right)+\bar{m}_{g, u} h_{g, u}\left(T_{u}\right) \\
& =v_{s} \rho_{s} \pi\left(\left(R_{b}+\delta\right)^{2}-R_{b}^{2}\right) h_{s}\left(T_{b}\right)+\bar{m}_{g, b} h_{g, b}\left(T_{b}\right) .
\end{aligned}
$$

Here, $\bar{m}_{g, u}=\left(v_{g} \rho_{g}\right)_{u} \pi R_{u}^{2}$ and $\bar{m}_{g, b}=\left(v_{g} \rho_{g}\right)_{b} \pi R_{b}^{2}$.

The amount of coal $\bar{m}_{s}^{\text {cons }}$ consumed in the appropriate control volume per unit time is given by

$$
\bar{m}_{s}^{\text {cons }}=v_{s} \rho_{s} \pi\left(R_{b}^{2}-R_{u}^{2}\right)=\bar{m}_{g, u}\left(\mu_{s}^{-}-\mu_{s}^{+}\right),
$$

where Eq. (35) has been used. Note that $\bar{m}_{s}^{\text {cons }}$ is always positive, since $v_{s}$ and $\left(R_{b}-R_{u}\right)$ are either both positive (reverse combustion) or both negative (forward combustion). The influx of hot coal to the control volume $\bar{m}_{s}^{\text {hot }}$ is given by

$$
\bar{m}_{s}^{\text {hot }}=-v_{s} \rho_{s} \pi\left(\left(R_{b}+\delta\right)^{2}-R_{b}^{2}\right) .
$$

Note that $\bar{m}_{s}^{\text {hot }}$ is negative for reverse combustion, since hot coal leaving the control volume in this case.

Now, we introduce the parameter $\beta$, which is the ratio of the hot coal influx to the amount of coal consumed per unit time. That is,

$$
\beta \equiv \frac{\bar{m}_{s}^{\text {hot }}}{\bar{m}_{s}^{\text {cons }}}=-\frac{v_{s} \rho_{s} \pi\left(\left(R_{b}+\delta\right)^{2}-R_{b}^{2}\right)}{v_{s} \rho_{s} \pi\left(R_{b}^{2}-R_{u}^{2}\right)} .
$$

The parameter $\beta$ is negative in the case of reverse combustion and positive otherwise. Note that the ranges $\beta<0,0<\beta<1$ and $\beta>1$ correspond to figures 2(a), 2(b) and 2(c), respectively. The value $\beta=1$ corresponds to the case, when $\left(R_{b}+\delta=R_{u}\right)$. Rigorously, the problem is not defined, when $\beta=0$. In this case, the flame speed is zero and coal enters the control volume from the radial direction. Eq. (39), being expressed in terms of $\beta$, takes the form

$$
(1-\beta) h_{s}\left(T_{u}\right)+\frac{1}{\mu_{s}^{-}-\mu_{s}^{+}} h_{g, u}\left(T_{u}\right)=-\beta h_{s}\left(T_{b}\right)+\frac{\mu_{g}^{+}}{\mu_{s}^{-}-\mu_{s}^{+}} h_{g, b}\left(T_{b}\right) .
$$

Here, we use Eqs. (35) and (40), recalling that $\mu_{g}^{-}=1$. Note that $\mu_{s}^{-}=\mu_{s}^{-}(\beta)$, while $\mu_{s}^{-}-\mu_{s}^{+}$is a constant determined by the stoichiometric conditions. It is important to note that Eq. (43), while being obtained for a cylindrical channel, is general and can be applied for channels of arbitrary geometry, as soon as $\bar{m}_{s}^{\text {hot }}$ is known.

Using Eq. (43), the burned temperature $T_{b}$ can be computed. The results of the calculations, which have been preformed for the conditions similar to those in the previous papers $[25,26]$, are given in Fig. 3(a). 

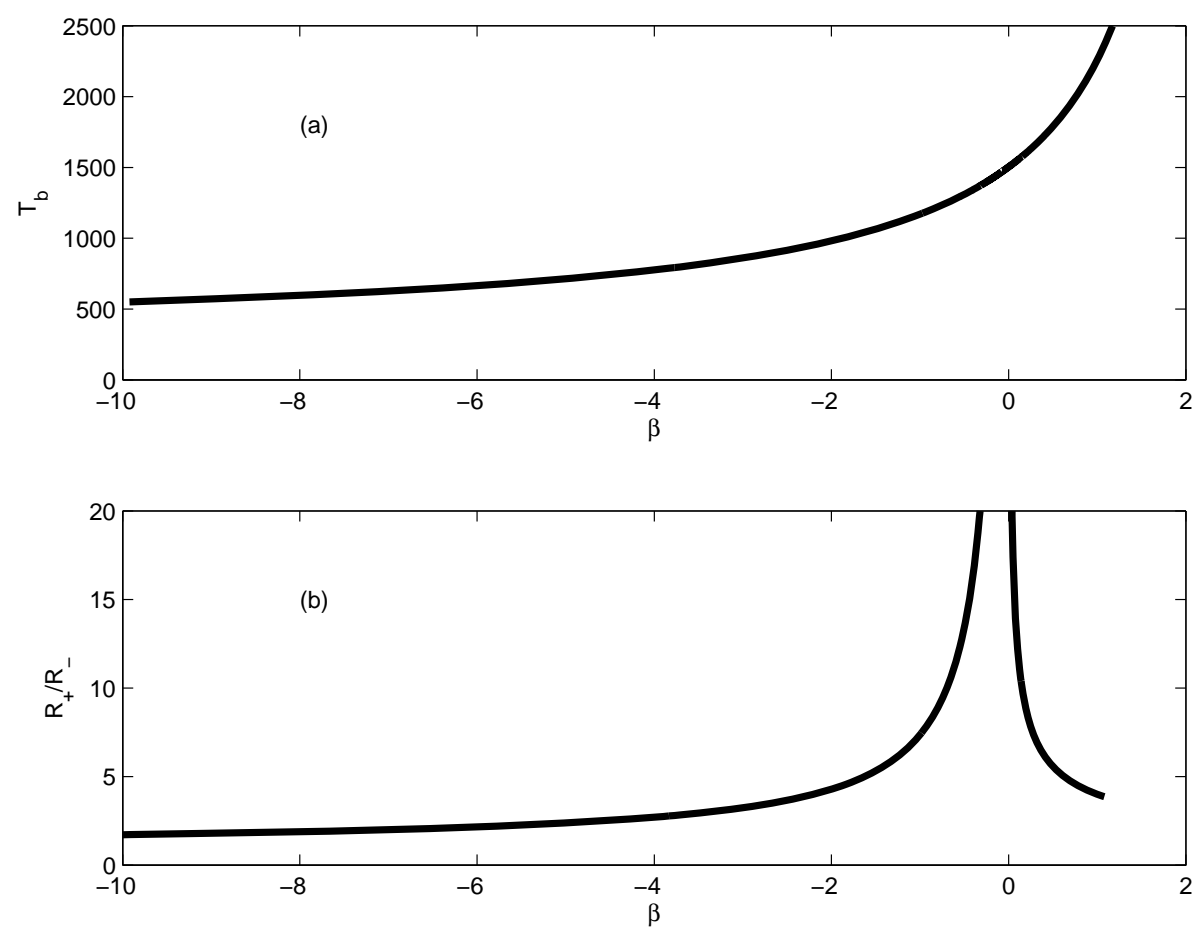

Figure 3: Parameters (a) the burned temperature $T_{b}$ and (b) the burned radius $R_{+}$as functions of the parameter $\beta$.

The value of $T_{b}$ increases with $\beta$ since less cold coal or more hot coal is delivered to the control volume at larger $\beta$. The temperature $T_{b}$ at $\beta=0$ corresponds to adiabatic temperature in stoichiometric conditions, while $T_{b}$ at $\beta=1$ is superadiabatic.

We introduce the initial radius of the channel $R_{-}=\min \left(R_{u}, R_{b}\right)$ and the radius after gasification $R_{+}=\max \left(R_{u}, R_{b}\right)$. In the case of the reverse combustion $R_{-}=R_{u}$ and $R_{+}=R_{b}$, while $R_{-}=R_{b}$ and $R_{+}=R_{u}$ for the forward combustion. The dependence of $R_{+}$on the parameter $\beta$ is presented in Fig. 3(b).

One can note that $R_{+}$becomes infinitely large at $\beta=0$ and this indicates that steady-state approach is not applicable to this case. The corresponding corrections to the theory are considered in the following sections.

\section{Primary physical factors determining the flame propagation speed.}

The velocity of the flame front $s$ is determined by Eq. (36) that after substitution of $S_{\text {cons }}=\pi\left(R_{+}^{2}-R_{-}^{2}\right)= \pm \pi\left(R_{u}^{2}-R_{b}^{2}\right)$ takes the form

$$
s=\frac{\mu_{s}^{-}-\mu_{s}^{+}}{\pi \rho_{s}\left(R_{u}^{2}-R_{b}^{2}\right)} \frac{Q_{h}\left(T_{b}, \ldots\right)}{H\left(\mu_{s}^{-}\right)}
$$


Fig. 4 shows the mass flow rate $M=\bar{m}_{g, u}$ of the injected air and the velocity of the flame front $s$ in a cylindrical gasification channel as a function of the parameter $\beta$. Positive values of the velocity mean that the flame front propagates downstream, while negative values imply that the front moves upstream.
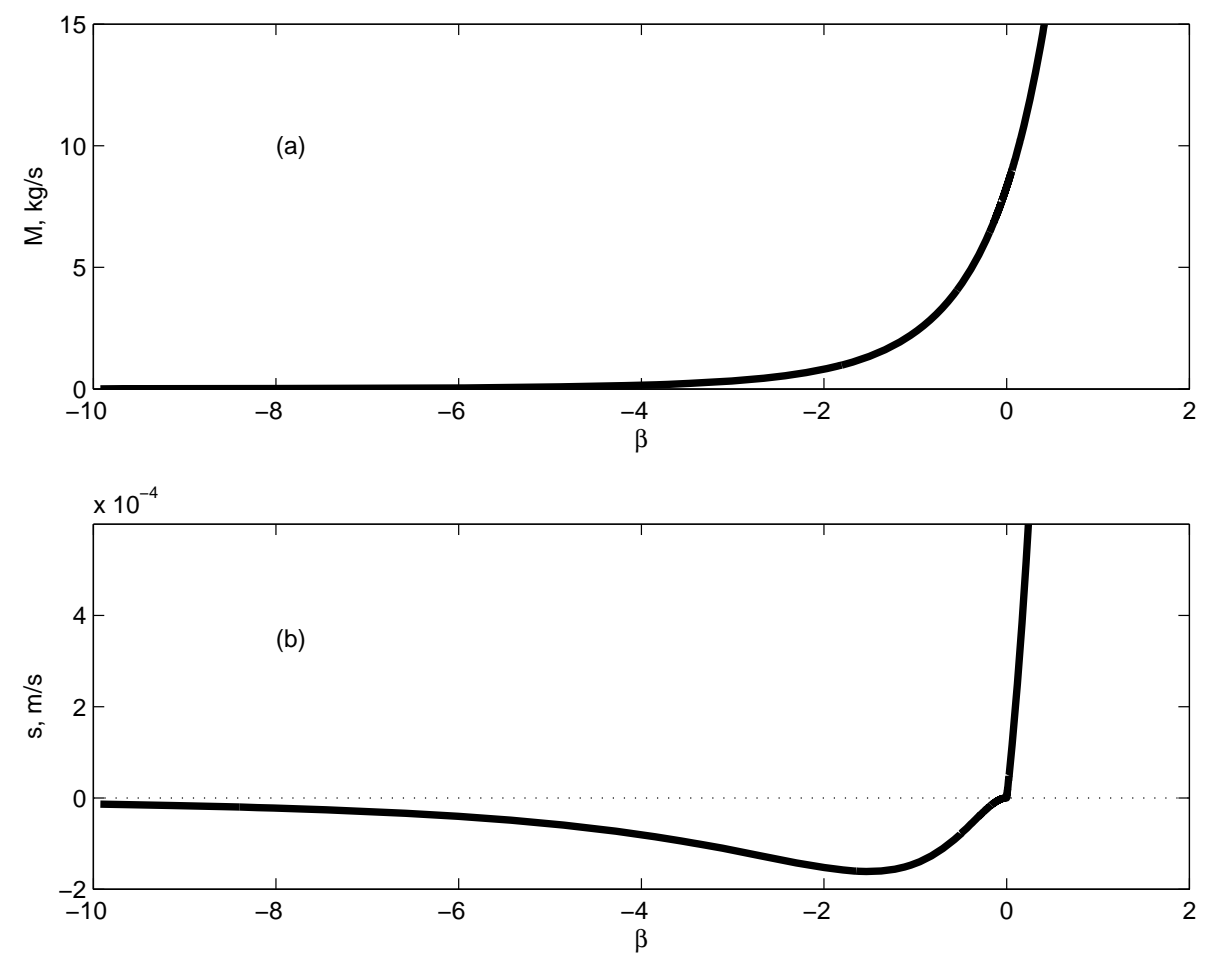

Figure 4: The mass flow rate of air (a) and the speed of the flame front (b) as functions of the parameter $\beta$.

The possibility of a flame propagation against the gas flow being slowed down by higher air velocity agrees with intuition. This intuition is based on the fact that, with increased $u$, the gas velocity relative to the flame $v_{g, u}=u-s$ can remain the same provided $s$ is increased (that is the absolute value of $s$ is decreased when $s$ is negative). In fact, $s$ is very small compared to $u$ and this effects is fully negligible. Practically, we do not need to distinguish $v_{g, u}$ and $u$. The same applies to the mass flow rates: the absolute mass flow rate of air $M$ is (nearly) the same as its relative rate $M=\bar{m}_{g, u}$. The flame speed is affected by the air flow rate trough energy balance in the channel.

The superadiabatic effect is induced by recycling the heat: coal is first heated by hot product gasses and then enters the combustion zone. Due to this effect, propagating downstream is thermodynamically beneficial for the flame, while the flame propagating upstream expends the heat on heating coal along its way. 
Higher temperatures in the combustion zone induce more intense reactions and increase the burning rate $Q_{h}$. A higher $M$ needs greater burning rate to sustain combustion. If the flame temperature is low, this flame can not move at an appreciable speed upstream since this would induce further heat losses. Very low temperatures and air flow rates correspond to practically extinct flames. As air flow increases, the flame temperature increases. This flame can now afford a heat loss associated with upstream motion and $s<0$ becomes substantial as shown in Fig. 4. (This figure is plotted for constant values of parameters $\bar{A}$ and $\left.\bar{\lambda}_{r}\right)$. A further increase in the air flow rate requires higher temperatures. This can be achieved only if the flame slows down or, at even higher flow rates, moves downstream.

As it can be seen from Eq. (32), the heat flux into the upstream cold region is higher for greater $Q_{h}$. If, due to a heat loss, the temperature of the flame is below the temperature, which is required for steady propagation, $Q_{h}$ and the heat flux into upstream regions is also smaller than required. The flame preheat zone cools down by the air stream causing oxygen to propagate further downstream. This either slows the flame propagation upstream or moves the flame downstream. As discussed previously, the flame temperature then increases compensating for the initial heat loss. A similar effect balances a possible overheat of the flame by propagating the flame upstream at a faster rate.

If the temperature of the flame is very high, the combustion becomes limited not only by the reaction rates but also by the rate of diffusion of oxidizer to the walls. This corresponds to large temperature gradients as compared to the channel width. As discussed in Section 3, the diffusion approximation of the radiant flux overestimates the value of the radian heat transfer when the temperature gradients are large. Thus, any further increase of the flame propagation speed is moderated by the rate of oxidizer diffusion and by the radiative heat transport. This effect is, to a certain extent, accounted for by introducing the parameter $K_{d}$ in Eq. (23). It should be noted that the whole solution based on using values averaged across the channel becomes inaccurate for very high reaction rates since, in this case, the temperature distribution is highly nonuniform across the channel. This case is specifically considered further in this work.

\section{Unsteady flame propagation.}

Figure 3 (b) indicates that $R_{+}$becomes infinite, when $\beta=0$. Since $R_{\mathrm{cv}} \geq$ $R_{+}$, we note that $s=0$ corresponds to $\beta=0$. Large $R_{+}$indicates that the theory based on a steady-state propagation of the flame becomes inapplicable when $\beta \rightarrow 0$. In a practical case, gasification starts in a channel of radius $R_{-}$and the initial channel burns not only in the axial but also in the radial direction. When combustion reaches the radius $R_{+}$, the flame can move steadily in the axial direction. If the air injection rate corresponds to $\beta=0$, then the radius $R_{+}=\infty$ can never be reached and a steady propagation is impossible. A similar restriction applies to small values of $\beta$ and large values of $R_{+}$. A steady solution exists in this case but remains impractical, since it would take a very 
long time to reach this solution. Thus, the developed theory based on axial propagation of the flame is practically limited only to the cases of sufficiently large absolute values of $\beta$, while radial propagation of the flame must be taken into account, when $\beta$ is small.

Let us assume that, at a given moment $t$, the current actual radius of the burned flame section is $R_{f+} \leq R_{+}$. Coal is consumed at the rate of $\bar{m}_{s}^{\text {cons }}=$ $\bar{m}_{s}^{-}-\bar{m}_{s}^{+}=s \rho_{s} S_{\text {cons }}$, where $S_{\text {cons }}=\pi\left(R_{+}^{2}-R_{-}^{2}\right)$ is the area of coal consumption in a steady flame. If $R_{f+}<R_{+}$, then the fraction $\gamma$ of $\bar{m}_{s}^{\text {cons }}$ is burned due to axial motion of the flame

$$
\bar{m}_{s}^{1}=s \rho_{s} \pi\left(R_{f+}^{2}-R_{-}^{2}\right)=\gamma \bar{m}_{s}^{\text {cons }}
$$

where

$$
\gamma=\frac{R_{f+}^{2}-R_{-}^{2}}{R_{+}^{2}-R_{-}^{2}}
$$

The remaining fraction $1-\gamma$ has to enter the combustion zone from other directions. Assuming the flame has the axial length of $L_{f}$ and the volume of $V_{f}=\pi R_{f+}^{2} L_{f}$ we obtain that $(1-\gamma) \bar{m}_{s}^{\text {cons }}=\rho_{s} d V_{f} / d t$. The value of $L_{f}$ is introduced as the overall length of the flame (involving oxidation and reduction zones) as can reasonably defined by the observer. This length is known to increase with $R_{f+}$ so we can put $L_{f}=C_{f} R_{f+}$. The constant $C_{f}$ is determined by the definition of the flame length and, as it is shown below, does not affect the outcomes. Since $d V_{f} / d t=3 \pi C_{f} R_{f+}^{2} \dot{R}_{f+}=3 \pi R_{f+}^{2} \dot{L}_{f}$ where $\dot{L}_{f}=d L_{f} / d t$ and $\dot{R}_{f+}=d R_{f+} / d t$ we can write $(1-\gamma) \bar{m}_{s}^{\text {cons }}=3 \pi R_{f+}^{2} \dot{L}_{f} \rho_{s}$. With introduction of the velocity of the downstream end of the flame $s_{b}=s+\dot{L}_{f}$, we obtain

$$
s_{b}=s+\frac{R_{+}^{2}-R_{f+}^{2}}{R_{+}^{2}-R_{-}^{2}} \frac{\bar{m}_{s}^{\mathrm{cons}}}{3 \pi R_{f+}^{2} \rho_{s}} .
$$

This equation determines the propagation speed of the downstream end of the flame which does not coincide with $s$ when $R_{f+}<R_{+}$and is the same as $s$ when $R_{f+}=R_{+}$. Here, we imply that $s$ does not change significantly due to the following arguments. If $R_{f+}<R_{+}$, coal enters the control volume from all directions. Coal that enters the volume in the radial direction has the same temperature as coal on the upstream side of the flame and this does not change the energy balance. Coal that enters the control volume from the downstream end has a high temperature but this additional energy is compensated by the increase of the total thermal energy within the control volume due to increase in the flame length. This allows us to keep the equation for $s$ without change as the first approximation. Finally, we consider what can happen if $R_{f+}>R_{+}$. In this case $R_{f+}$ can not decrease with time and a narrower channel, which has radius $R_{+}$, is burned into the virgin coal surrounding the channel of radius $R_{-}$, while a wider cavity of radius $R_{f+}$ remains unaffected.

The effective kinetic parameters are now evaluated

$$
\bar{\lambda}_{r}=\frac{32}{3} \pi R_{f, u}^{3} \sigma T_{b}^{3}, \quad \bar{A}=A R_{f+}
$$




$$
T_{d}=-\frac{T_{E}}{\ln \left(\frac{M}{\rho_{g} A \Delta x_{d}}\right)}, \quad \Delta x_{d} \equiv \frac{M}{\rho_{g} \bar{A} K_{d}}=C_{d} R_{-},
$$

where $A$ is constant and $R_{f, u}=R_{-}$for reverse combustion and $R_{f, u}=R_{f+}$ for forward combustion. Here, we use that the heterogeneous reaction rate is proportional to the surface of the wall at a given $x$-location. Values of the effective kinetic parameters $A$ and $T_{E}$ depend on coal properties. The integral of the effective conductivity across the flow is determined with the use of Eq. (6) and using $T_{b}$ as a characteristic temperature (while this approach is conventional for kinetically limited flames, the wall temperature is close to $T_{b}$ for diffusion-limited flames). The value $\Delta x_{d}$ has the dimension of length and can be interpreted as the characteristic length of leading section of the flame which is responsible for the radiative heat flux directed upstream. Since $R_{-}$is the only parameter of the channel (before burning takes place) that has the dimension of length and can affect $\Delta x_{d}$ we put $\Delta x_{d}=C_{d} R_{-}$where $C_{d}$ is constant. In the kinetically limited regime $\Delta x_{d}$ would be the length of the preheat zone but here we are interested in the diffusion-limited case, since this case is linked to the parameter $K_{d}$. At the limit of fast kinetics and slow diffusion, the leading zone is formed by areas of high temperature on the walls, while the air stream is still cold in the core of the flow. According to the analysis of Section 3, the maximal radiative heat flux corresponds to the effective length of disturbance given by $\Delta x=\frac{4}{3} R$ and we put $C_{d}=4 / 3$. Note that in any case $\Delta x_{d}$ can not be greater than the length of the oxygen zone $\Delta x_{\mathrm{O} 2}$ (that is the sum of preheat and reaction zones, where oxygen is present). Since $\Delta x_{\mathrm{O} 2} \sim 40 R$ this imposes the upper limit on possible values for $C_{d}$. In fact, when reactions are fast, $\Delta x_{d}$ is much smaller than $\Delta x_{\mathrm{O} 2}$ : oxygen is present in the core of the flow in the same cross-sections where wall temperatures are already very high.

The premixed-type flames are characterized by balance of reaction, heat conduction and heat convection - the terms characterizing all three processes are of the same order. Premixed combustion is impossible if one of these terms is negligible everywhere in the flow. The length of the reaction zone $\Delta x_{r}$ can be estimated by diffusion/reaction balance of the heat

$$
\frac{\partial}{\partial x}\left(\bar{\lambda} \frac{\partial T}{\partial x}\right) \sim \bar{W}_{h} \sim R_{f+} A K(T) .
$$

Considering that $\bar{\lambda} \sim R_{f+}^{3}$ and $\bar{W}_{h} \sim R_{f+} A$, we obtain $\Delta x_{r} \sim\left(\bar{\lambda} / \bar{W}_{h}\right)^{1 / 2} \sim$ $R_{f+}$. This gives a justification for assuming $L_{f} \sim R_{f+}$ in the reaction-limited regime. For the diffusion-limited regime, this estimate follows from basic properties of turbulence discussed in the next section.

\section{Comparison with experimental observations}

The suggested theory is in agreement with qualitative observations by Chernyshev [8], who noted that if the air injected into a gasification channel at a low speed, the flame tend to propagate towards the injection point but, if the air rate 

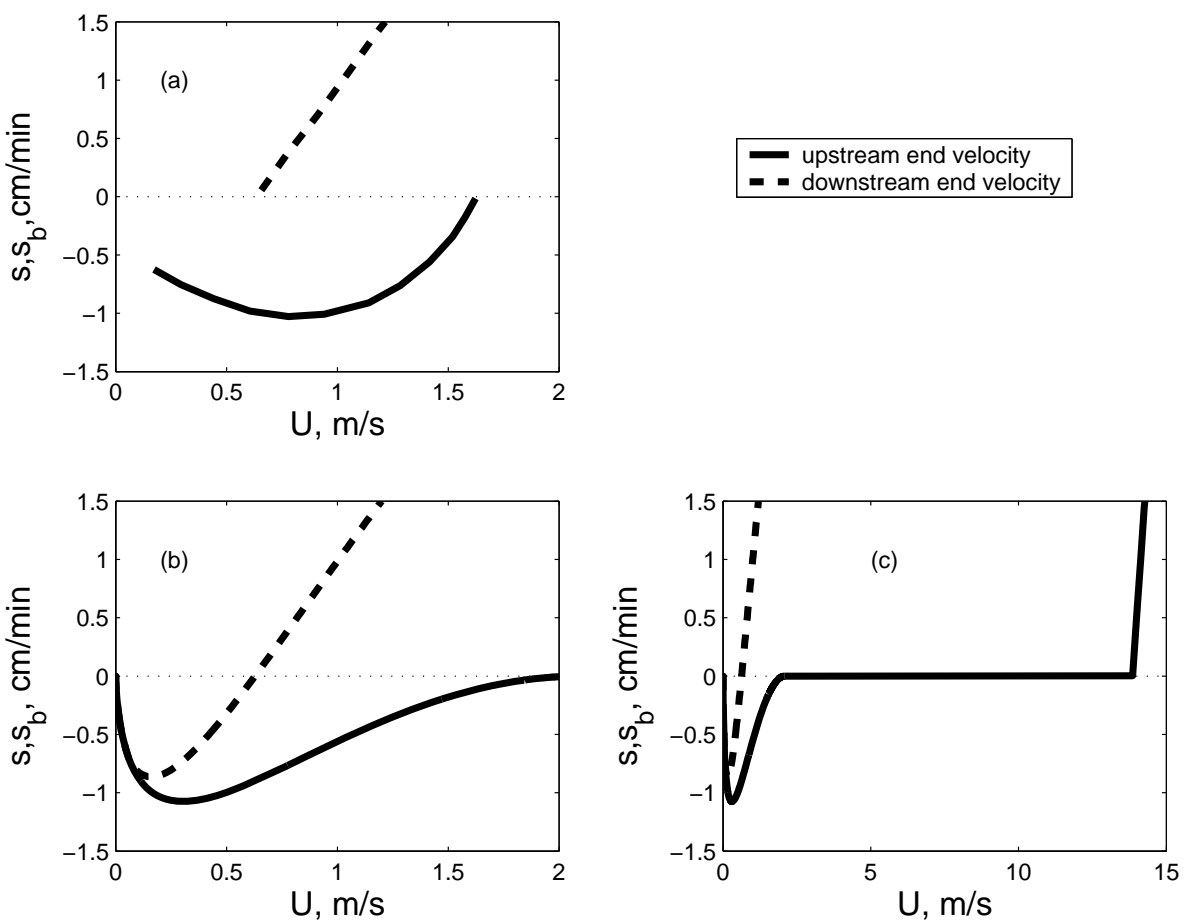

Figure 5: Dependence of a burn velocity on the air flow rate: (a) Data from Skafa [9], (b) and (c) Present theory, plotted with with different limits

increases, the cavity tend to grow in the downstream direction. It is also known that flame propagation is faster when oxygen is used instead of air[9]. This behavior is also expected since oxygen-fed flames are hotter and have higher reaction rates. We now perform a more quantitative comparison with experiments. Fig. 5(a) demonstrates two burn velocities $s$ and $s_{b}$ as function of the injection air speed $u_{0}=M /\left(\pi R_{-}^{2}\right)$ in the upstream undisturbed section of the channel. These results are reproduced from the book by Skafa [9], although we changed the sign of $s$ in agreement with notations that we use in the present work. Fig. 5(b) presents velocities $s$ and $s_{b}$ obtained from Eqs. (44) and (47) for the following values of the model parameters: $A=1.3 \mathrm{e} 4 \mathrm{~s}^{-1}, T_{E}=7700 \mathrm{~K}, T_{u}=300 \mathrm{~K}$, $R_{-}=0.5 \mathrm{~m}, R_{f+} / R_{-}=2.5$. If $u_{0}$ tends to zero and no air is supplied, this extinguishes the flame and $s$ tends to zero as well. Qualitative agreement between these figures is obvious, although the theory predicts the possibility of negative values for $s_{b}$ and positive values for $s$ which is not shown in Fig.5(a). While existence of negative $s_{b}$ is physically obvious - the velocity of the downstream end of the flame is not likely to jump from negative $s$ to zero for a minor change in the air speed, the possibility of moving the whole flame in the direction of the flow, which corresponds to positive $s$, warrants a special discussion. 
One can note that as $s$ approaches zero from negative side, $s$ remains small for a range of air velocities (Fig. 5(b)). While the upstream end of the flame remains steady, the channel grows in the radial direction and also increases its length. If the air speed is increased further, can the upstream end of the flame start moving in the forward direction? Physically, this is possible only if the air flow cools the upstream end of the flame and flame has to retreat in the direction of the air stream were unburned coal is hot and this restores the heat balance. This can happen if the flame is controlled by the reaction rates (the flame is not very hot) and cooling effect of the air flow is strong. This slows down the reaction and oxygen penetrates further downstream. Effectively the flame is locally extinguished by the flow upstream and reestablishes itself in a hotter place downstream. Fig. 5(c) presents the same plot as 5(b) but using extended limits. Note that this figure indicates the possibility of positive $s$ for very high injection rates.

The situation is, however, different, if the flame is very hot and controlled by diffusion. We consider the limit of high temperatures, high activation energy and a strong air flow. Under these conditions, the surface of the channel has two zones cold and hot. The temperature is insufficiently high in the cold zone to initiate reactions, while in the hot zone any oxygen on the surface reacts instantly. Since the activation energy is high, these zones are separated only by a very small distance. The overall reaction rate is determined by the rate of diffusion of oxygen to the hot zone, while the oxygen concentration on hot walls is essentially zero. The temperature is very high near the surface and is much lower in the middle of the air stream. Rigorously, averaging of the flow parameters across the channel is not applicable to this regime. The flow in a gasification channel is turbulent, otherwise laminar diffusion would not be able to deliver oxygen at appreciable rates. If the flow rate is increased by the factor of 2 , the average parameters of this flow remain the same since largescale structures of turbulence are invariant with respect to changes in Reynolds number. In simple terms, the turbulent diffusion coefficient is also doubled. The walls are rough and this eliminates any viscous sublayer effects on the flow. Hence the flame position does not change under these conditions and does not move downstream, although the overall burning velocity increases. Similarly, if the air velocity is decreased twice the flame does not move upstream (although a large reduction in velocity can result in attenuation of turbulence and this would affect the flow). Hence, for this limit of a turbulent flame fully controlled by diffusion, there is no control over the flame position by means of changing the injection rate. Combustion of a real coal starts from devolitalization reactions at low temperatures and can be cooled by the air stream. If these reactions play a noticeable role in initiating the rest of oxidation process or in the overall energy balance, the flame position is affected by the air speed and becomes controllable.

When the flame is ignited, $R_{f+}$ is approximately the same as $R_{-}$. The present theory predicts positive $s_{b}$ and negative $s$ for a wide range of $u_{0}$. This initial trend of the flame to propagate in all directions was noted by Skafa [9]. Since the channel walls downstream are initially cold, this imposes an additional 
energy penalty, which is determined by the need to heat the expanding flame area, and the reaction rates are lower than under developed conditions.

\section{Conclusions}

A theoretical model, which describes propagation of the flame front through a channel with reacting walls, is suggested in the present study. Simplifying assumptions are made to allow for compact analytical representation of the results, while retaining main physical effects that are of primary importance to the phenomena in question. The suggested theory, which is based on the analysis of energy balance in a gasification channel, explains the major physical factors that are responsible for the flame speed and is in a qualitative agreement with experimental observations obtained in practical UCG operations.

\section{Acknowledgement}

The work has been supported by the Australian Research Council. Part of this work has been done during the sabbatical visit of the second author to The University of Queensland supported by University of Wyoming and the Australian Research Council. The authors thank D.A. Klimenko for programming assistance.

\section{References}

[1] T. Von Karman, G. Millan, Theoretical and experimental studies on laminar combustion and detonation, in: Fourth Symposium (International) on Combustion, The Combustion Institute, Pittsburg, 1953, pp. 173-177.

[2] G. F. Carrier, F. E. Fendell, P. S. Feldman, A comparison of one and two dimensional flame quenching: Heat transfer results, Proceedings of the Combustion Institute 20 (1984) 67-74.

[3] F. Benkhaldoun, B. Larrouturou, Explicit adaptive calculations of wrinkled flame propagation, International Journal for Numerical Methods in Fluids 7 (11) (1987) 1147-1158.

[4] A. Y. Klimenko, Examining the cascade hypothesis for turbulent premixed combustion, Combust.Sci. and Tech. 139 (1998) 15-40.

[5] V. Akkerman, V. Bychkov, L.-E. Eriksson, Numerical study of turbulent flame velocity, Combustion and Flame 151 (2007) 452-471.

[6] S. Chakraborty, A. Mukhopadhyay, S. Sen, Interaction of Lewis number and heat loss effects for a laminar premixed flame propagating in a channel, International Journal of Thermal Sciences 47 (2008) 84-92.

[7] A. G. Class, B. J. Matkowsky, A. Y. Klimenko, A unified model of flames as gasdynamic discontinuities, J.Fluid Mech 491 (2003) 11-49. 
[8] A. B. Chernyshev, Izbrannye Trudy, Izdatel'stvo AN USSR, Moscow, 1956.

[9] P. V. Skafa, Underground Coal Gasification, Gosgortechizdat, Moscow, 1960.

[10] G. J. Hurloff, Underground coal gasification cavity growth model, Journal of Energy 7 (1983) 410-415.

[11] K. Y. Park, T. F. Edgar, Modeling of early cavity growth for underground coal gasification, Industrial \& Engineering Chemistry Research 26 (1987) $237-246$.

[12] L. Yand, S. Liu, Numerical simulation on heat and mass transfer in the process of underground coal gasification, Numerical Heat Transfer Part A $44(2003) 537-557$.

[13] L. Yang, J. Liang, L. Yu, Clean coal technology - Study on the pilot project experiment of underground coal gasification, Energy 28 (2003) 1445-1460.

[14] L. Yand, Study on the model experiment and numerical simulation for underground coal gasification, Fuel 83 (2004) 573-584.

[15] M. J. Prins, K. J. Ptasinski, Energy and exergy analyses of the oxidation and gasification of carbon, Energy 30 (2005) 982-1002.

[16] K. Yamashita, L. Barreto, Energyplexes for the 21st century: Coal gasification for co-producing hydrogen, electricity and liquid fuels, Energy 30 (2005) 2453-2473.

[17] G. Perkins, V. Sahajwalla, A numerical study of the effects of operating conditions and coal properties on cavity growth in underground coal gasification, Energy \& Fuels 20 (2006) 596-608.

[18] G. Perkins, V. Sahajwalla, Modelling of heat and mass transport phenomena and chemical reaction in underground coal gasification, Chemical Engineering Research and Design 85 (2007) 329-343.

[19] A. Khadse, M. Qayyumi, S. Mahajani, P. Aghalayam, Underground coal gasification: A new clean coal utilization technique for India, Energy 32 (2007) 2061-2071.

[20] M. S. Blinderman, R. M. Jones, The chinchilla IGCC project to date: Underground coal gasification and environment, in: Gasification Technologies Conference, San Francisco, USA, October 27-30, 2002.

[21] M. S. Blinderman, B. Anderson, Uderground coal gasification for power generation: Efficiency and $\mathrm{CO}_{2}$-emissions, in: $12^{\text {th }}$ International Conference on Coal Science, Cairns, Australia, November 2-6, 2003, paper No. $13 \mathrm{C} 1$. 
[22] O.A.Plumb, A. Y. Klimenko, The stability of evaporating fronts in porous media, J. Porous MediaTo appear.

[23] H. Hottel, J. Keller, Effect of reradiation on heat transmission in furnaces and through openings, Trans. ASME 55, IS-55-6 (1933) 39-49.

[24] J. Howell, A catalog of radiation view factors, Tech. rep., http://www.me.utexas.edu/ howell/ (accessed online 2008).

[25] M. S. Blinderman, D. N. Saulov, A. Y. Klimenko, Forward and reverse combustion linking in underground coal gasification, Energy 33 (2008) 446454.

[26] M. S. Blinderman, A. Klimenko, Theory of reverse combustion linking, Combustion and Flame 150 (2007) 232-245.

[27] Y. B. Zeldovich, G. I. Barenblatt, V. B. Librovich, G. M. Makhviladze, The mathematical theory of combustion and explosions, Consultants Bureau, 1985.

[28] A. G. Class, B. J. Matkowsky, A. Y. Klimenko, Stability of planar flames as gasdynamic discontinuities, J.Fluid Mech 491 (2003) 51-63. 